\section{OPEN JOURNAL SYSTEMS}

ISSN:2237-2202
Available on line at Directory of Open Access Journals

Journal of Hyperspectral Remote Sensing v.7, n.7 (2017) 432-438

www.periodicos.ufpe.br/revistas/jhrs
Journal of

Hyperspectral

Remote Sensing

\title{
Multivariate analysis of soil moisture data
}

\author{
Any G. de Sena*, Josiclêda D. Galvincio**, Valéria S. de O. Costa***, Rodrigo de Q. Miranda****, Maria do Socorro \\ B. de Araújo ${ }^{* * * * *}$ Magna Soelma Beserra de Moura****** \\ "Mestranda do Programa de Pós-Graduação de Desenvolvimento e Meio Ambiente - PRODEMA, Universidade Federal de \\ Pernambuco -UFPE, Recife, Pernambuco, Brasil. E-mail: any.sena.geo@ hotmail.com (Corresponding author) \\ ${ }^{* *}$ Professora e Pesquisadora do Departamento de Ciências Geográficas e do PRODEMA/UFPE, Recife, Pernambuco, Brasil. \\ E-mail: josicleda@gmail.com \\ **** Professora, CAPES/PNPD-PRODEMA/UFPE, Recife, Pernambuco, Brasil. E-mail: costavso@ yahoo.com.br. \\ *****B Bolsista CAPES/PNPD/FACEPE/ PRODEMA, Recife, Pernambuco, Brasil. E-mail: rodrigo.qmiranda@gmail.com \\ *Professora e Pesquisadora do Departamento de Ciências Geográficas e do PRODEMA/UFPE, Recife, Pernambuco, Brasil. \\ E-mail: socorro@ufpe.br \\ ******** Pesquisadora da EMBRAPA semiárido. E-mail: magna.moura@embrapa.br
}

Received 13 June 2017; accepted 30 October 2017

\begin{abstract}
Soil water content is an important variable in the understanding of hydrology in agricultural and environmental systems in a region. It is known that soil moisture is related to soil characteristics, porosity, depth, hydraulic conductivity, among others, that is, characteristics that define its typology. Studies related to soil moisture are still very precarious in Brazil. Recently, the Europe Space Agency has provided soil moisture data estimated worldwide with satellite data. This availability made possible the spatial and temporal assessment of soil moisture for different studies in the world, even though we did not know the accuracy of these data. Many studies have used multivariate analysis to find groups that have similar characteristics that can be analyzed and managed with the same actions. Therefore, this study sought to analyze the similarities and dissimilarities between soil types when considering the characteristics of soil moisture, precipitation, soil elevation and soil depth. After applying the statistical methods it was possible to perceive that the soil moisture does not depend strongly on the precipitation and to suggest caution in the analysis of the relations between the humidity factor and the others scored.

Keywords: multivariate statistics, Ward method, soil moisture.
\end{abstract}

\section{Análise multivariada de dados de umidade do solo}

\section{RESUMO}

O conteúdo de água no solo é uma variável importante na compreensão da hidrologia em sistemas agrícolas e ambientais de uma região. Sabe-se que a umidade do solo tem relação com as características do solo, porosidade, profundidade, condutividade hidráulica, dentre outros, ou seja, características que define sua tipologia. Os estudos relacionados a umidade do solo ainda são bastante precários no Brasil. Recentemente, a Europe Space Agency disponibilizou dados de umidade do solo para todo o mundo estimados com dados de satélites. Está disponibilização tornou possível a avaliação espacial e temporal de umidade do solo para diferentes estudos do mundo, mesmo não sabendo a precisão destes dados. Muitos estudos têm se utilizados de analise multivariada para encontrar grupos que possuem características semelhantes que possam ser analisados e gerenciados com as mesmas ações. Portanto este estudo buscou-se analisar as similaridades e dissimilaridades entre os tipos de solos quando considerado as características umidade do solo, precipitação, elevação do terreno e profundidade do solo. Após a aplicação dos métodos estatísticos foi possível perceber que a umidade do solo não depende fortemente da precipitação e sugerir cautela nas análises de relações entre o fator umidade e demais pontuados.

Palavras-chave: estatística multivariada, método de Ward, umidade do solo.

\section{INTRODUÇÃO}

A água é necessária para a existência e manutenção humana assim como influência de direta ou indireta dos principais fenômenos que ocorrem nos solos. O intemperismo, os processos de formação, atividade biológica, crescimento de plantas, assim como, poluição do lençol freático recebem impacto 
direto do regime hídrico dos solos. O conteúdo de água no solo é uma variável importante na compreensão da hidrologia em sistemas agrícolas e ambientais de uma região (White, 2005), principalmente semiárida, uma vez que está prática é dependente da disponibilidade deste recurso. Consequentemente, as práticas agrícolas necessitam de estratégias para o desenvolvimento de culturas com maior precisão, ou seja, estratégias de gerenciamento com o potencial de integrar conjuntos de dados variados através de informações e tecnologias no desenvolvimento de decisões e de produção de cultura Whiting (2006). Uma vez que as novas tecnologias contribuem para o aumento de produtividade das áreas agrícolas, gerando dados que podem promover o aumento da eficiência e redução dos custos da produção e os impactos sobre o ambiente.

Compreender as dinâmicas de disponibilidade hídrica no solo e sua variação no tempo e no espaço, é uma atividade que exige atenção a fatores biofísicos terrestres e atmosféricos. A umidade do solo é um recurso considerado limitante, pois sua variabilidade pode ser atribuída a alguns fatores, a exemplo o índice pluviométrico, tipologia e profundidade do solo, a declividade do terreno e o uso da terra. $\mathrm{O}$ teor de água no solo é de extrema importância para o desenvolvimento da cobertura vegetal, pois é um dado que corrobora diretamente com as estratégias de gerenciamento agrícola e gestão ambiental.

Os estudos relacionados a umidade do solo ainda são bastante precários no Brasil. Recentemente, a Europe Space Agency disponibilizou dados de umidade do solo para todo o mundo estimados com dados de satélites, http://rs.geo.tuwien.ac.at/dataviewers/. Está disponibilização tornou possível a avaliação espacial e temporal de umidade do solo para diferentes estudos do mundo, mesmo não sabendo a precisão destes dados.

Sabe-se que a umidade do solo tem relação com as características do solo, porosidade, profundidade, condutividade hidráulica, dentre outros, ou seja, características que definem o tipo de solo. Sabe-se também que solos com características físicas semelhantes podem apresentar diferentes umidades.
Muitos estudos têm se utilizados de analise multivariada para encontrar grupos que possuem características semelhantes que possam ser analisados e gerenciados com as mesmas ações.

Segundo Sartori (2008) as multivariadas são modelos estatísticos que consideram muitas variáveis ao mesmo tempo, o que demanda um exame detalhado e rigoroso dos dados, pois o tratamento inadequado pode ter efeitos "catastróficos".

Diante do exposto, o objetivo deste estudo foi analisar as similaridades e dissimilaridades entre os tipos de solos quando considerado as características umidade do solo, precipitação, elevação do terreno e profundidade do solo.

\section{Material e métodos}

\section{Caracterização da área de estudo}

$\mathrm{O}$ estudo foi desenvolvido no âmbito dos municípios: Goiana-PE; Gado Bravo-PB; Serra Branca-PB; São José do Egito-PE; Princesa IsabelPB; Ibiara-PB; Jardim-CE; Ipubi-PE; Araripina-PE; e Belém do Piauí-PI, representando quatro estados do Nordeste do Brasil.

\section{Aquisição dos dados}

As variáveis "tipos de solo" e "profundidade", foram obtidas no manual de solos do Brasil EMBRAPA (2006), em que: PV - Argissolos Vermelhos, PVA- Argissolos Vermelho-Amarelo LVA- Latossolos Vermelho-Amarelo, e TC Luvissolos Crômicos.

A variável "Elevação do terreno" foi obtida através do Google Earth Pro. Os dados de "Precipitação diária" foram obtidos através da Agência Executiva de Gestão das Águas (AESA) e Agência Pernambucana de Águas e Clima (APAC), para os estados da Paraíba e Pernambuco, respectivamente. Os dados de Precipitação referentes ao município de Jardim- CE e Belém do Piauí foram preenchidos com dados da estação meteorológica mais próxima do município, mas pertencente ao Estado de Pernambuco. Foram utilizados neste estudo dados do período de 1 a 16 de janeiro de 1980. Os dados foram organizados em planilha Excel (Figura 1).

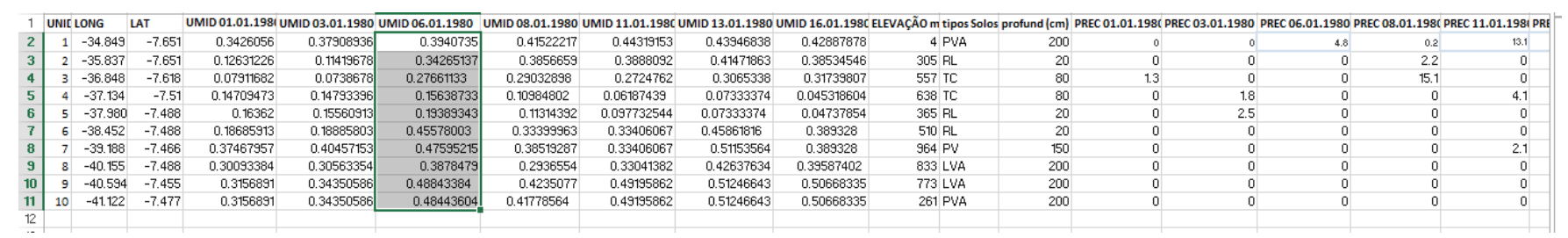

Figura 1 - Organização dos dados obtidos na planilha do Excell.

Na obtenção dos dados de umidade do solo, foram escolhidos pontos aleatórios, visando cobrir de leste-oeste as microrregiões do Nordeste Brasileiro. As informações foram obtidas na plataforma Climate 
Change

Initiative,

link

http://rs.geo.tuwien.ac.at/cci_dataviewer/, que disponibiliza dados globais de umidade do solo em diversas resoluções. Logo, para esta ocasião os dados foram obtidos numa resolução de 100 por $100 \mathrm{~m}$; camada $(0-7 \mathrm{~cm})$. Todos os dados são datados de $1 \mathrm{a}$ 16 de Janeiro de 1980 (Figura 2). A escolha dos dados neste período ocorreu na tentativa de escolher um período com menor ação antrópica e representar uma situação sem interferência das mudanças climáticas atuais. $\mathrm{O}$ intuito é identificar as relações existentes entre a precipitação e umidade do solo com menor interferência humana e das mudanças climáticas.

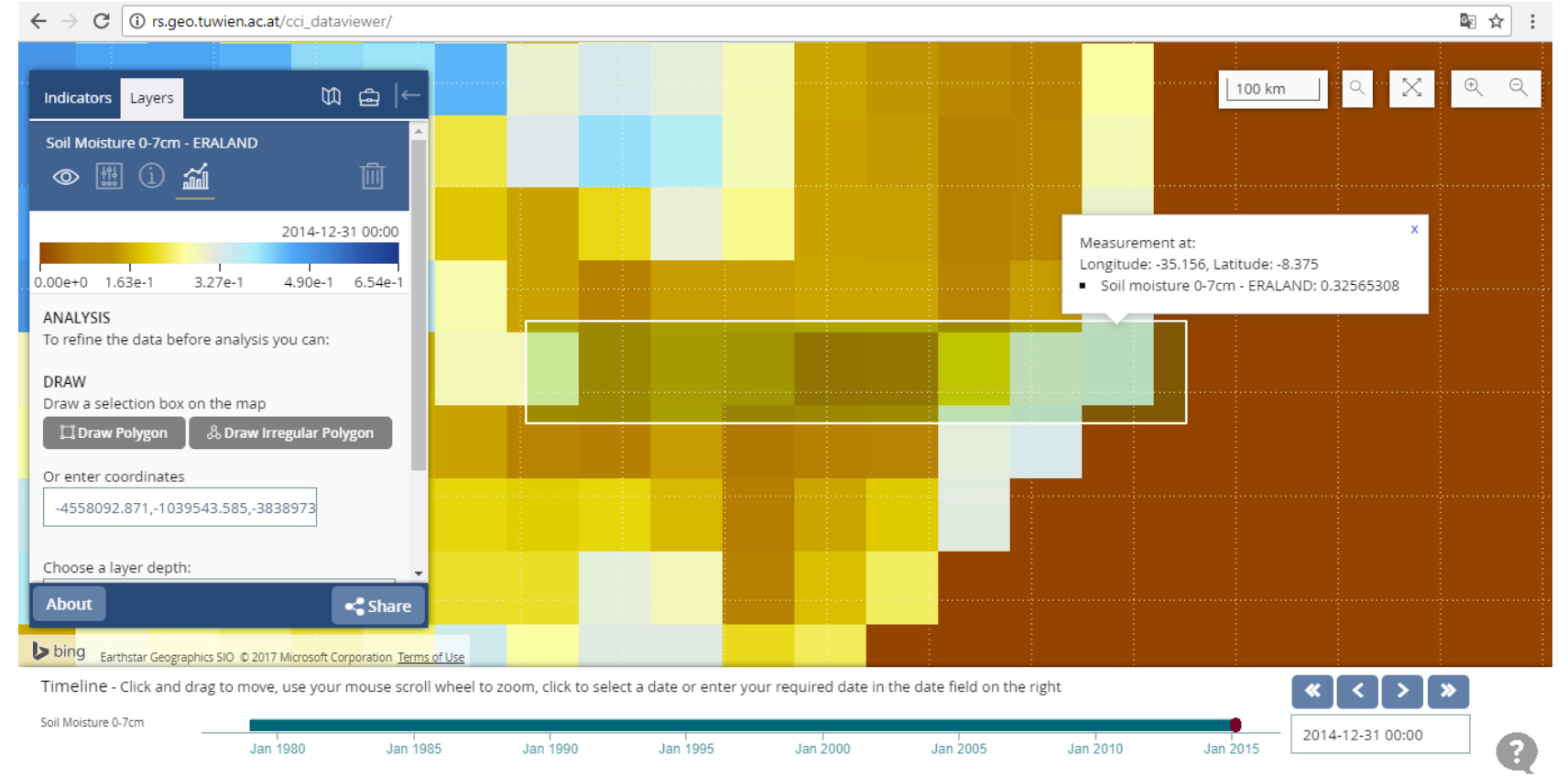

Figura 2 - Plataforma Climate Change Initiative

\section{Análise estatística}

Em Software estatístico foi aplicado o método de Clusterização hierárquica, através do método iterativo de Ward e a análise do índice de Distância euclidiana que posteriormente possibilitou a análise da distância mínima e máxima entre os valores apresentados.

\section{Método de Ward}

Segundo Hair et al. (2005), o método de Ward consiste em um procedimento de agrupamento hierárquico no qual a medida de similaridade usada para juntar agrupamentos é calculada como a soma de quadrados entre os dois agrupamentos feita sobre todas as variáveis. Esse método tende a resultar em agrupamentos de tamanhos aproximadamente iguais devido a sua minimização de variação interna. Em cada estágio, combinam-se os dois agrupamentos que apresentarem menor aumento na soma global de quadrados dentro dos agrupamentos.

Outros detalhes deste método podem ser encontrados em Fechine e Galvíncio (2008).

\section{Resultados e discussão}

Inicialmente foi realizado a avaliação dos solos que apresentavam características similares de umidade do solo, profundidade, precipitação e elevação do terreno. Foi obtido quatro grupos homogêneos (Figura 3). Grupo 1: contou com três tipos de solos, sendo PV (ponto 7), LVA (ponto 8), PVA (ponto 9) e PVA (ponto 10); Grupo 2: PVA (ponto 1); Grupo 3: TC (ponto 4), RL (ponto 5) e RL (ponto 6); Grupo 4: TC (ponto 3). Nota-se que o PVA (ponto 10) não se juntou com o PVA (ponto 1) e nem o TC (ponto 4) com o TC (ponto 3). Para o PVA o que diferenciou foi a umidade do solo que no ponto 10 estavam sempre superiores ao ponto 1 , mesmo no ponto 10 não ocorrendo precipitação. Isso nos sugere uma área de agricultura irrigada no ponto 10 . No caso da TC a umidade está relacionada a precipitação, uma vez que no ponto 3 onde choveu mais a umidade do solo estavam sempre mais altas. Sugere que o estudo da relação entre precipitação e umidade do solo para grandes áreas geográficas deve ser feito com cautela uma vez que algumas áreas irrigadas apresentam maiores umidades do solo que não estão relacionadas a precipitação. Por outro lado, o estudo da umidade do solo para avaliação dos recursos hídricos de uma bacia hidrográfica é mais real uma vez que o uso antrópico do solo para agricultura irrigada irá interferir na umidade do solo sem a presença de precipitação e essa umidade irá interferir no manejo e planejamento dos recursos hídricos da bacia hidrográfica. 


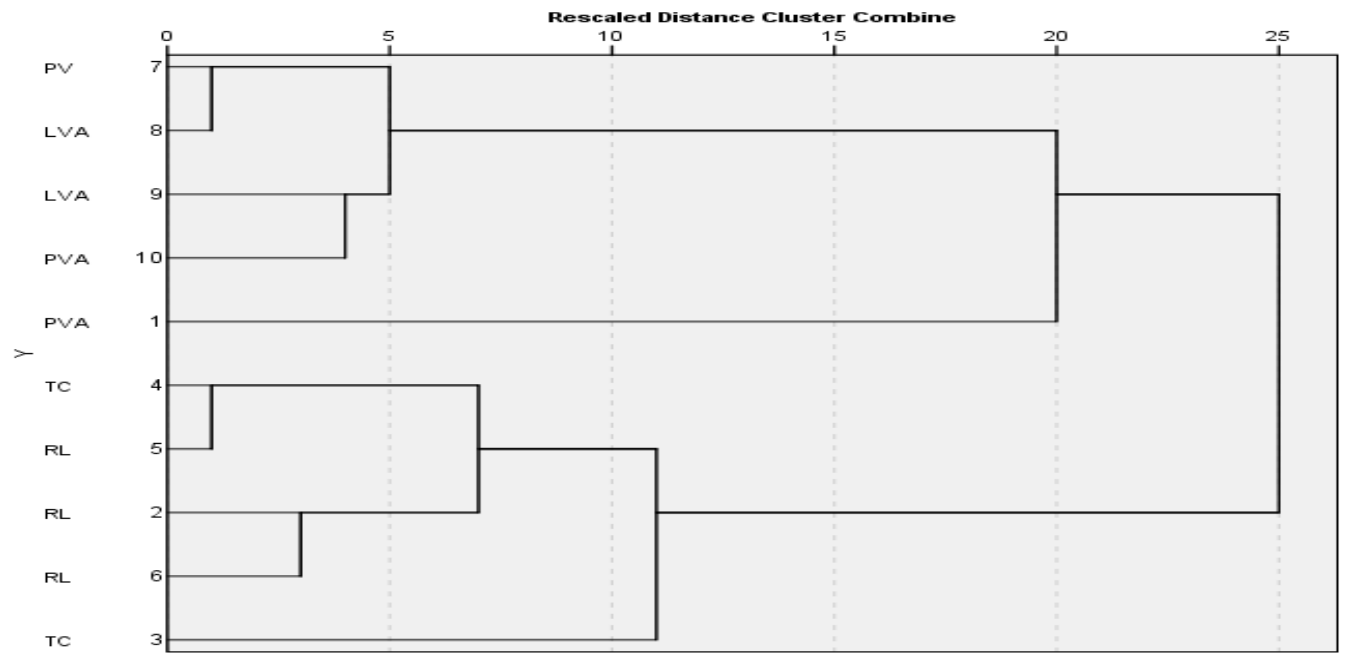

Figura 3 - Dendrograma pelo método de Ward relacionados a umidade do solo, precipitação, elevação do terreno e profundidade do solo. PV (Argissolos Vermelhos), LVA (Latossolos Vermelho-Amarelo), PVA (Argissolos Vermelho-Amarelo), TC (Luvissolos Crômicos) e RL (Neossolos Litólicos).

Para verificar o quanto distante estão o PVA (ponto 10) e o PVA (ponto 1), como também TC (ponto 3) e o TC (ponto 4) analisou a matriz de proximidade pela distância euclidiana ao quadrado, Tabela 1. Nota-se que os TCs apresentaram menores distâncias quando comparado com os PVAs, isso porque os TCs são pontos que estão separados devido a variação na precipitação, mas o PVAs está separado pela umidade do solo que possuem características externas aos dados analisados.

Tabela 1 - Matriz de proximidade pela distância euclidiana ao quadrado.

\begin{tabular}{|c|c|c|c|c|c|c|c|c|}
\hline \multirow[t]{2}{*}{ Dados } & \multicolumn{8}{|c|}{ Distância Euclidiana Quadrada } \\
\hline & 1:PVA ${ }^{1}$ & $2: R L^{2}$ & 3:TC $\mathrm{TC}^{3}$ & 4:TC & 5:RL & 6:RL & $7: \mathbf{P V}^{4}$ & 8:LVA ${ }^{5}$ \\
\hline 1:PVA & .000 & 49.506 & 88.381 & 63.500 & 74.928 & 64.473 & 48.203 & 52.553 \\
\hline $2: \mathrm{RL}$ & 49.506 & .000 & 28.215 & 24.505 & 20.915 & 12.327 & 24.565 & 17.960 \\
\hline 3:TC & 88.381 & 28.215 & .000 & 33.454 & 35.596 & 30.141 & 52.038 & 40.080 \\
\hline 4:TC & 63.500 & 24.505 & 33.454 & .000 & 5.384 & 15.632 & 30.997 & 23.587 \\
\hline $5: R L$ & 74.928 & 20.915 & 35.596 & 5.384 & .000 & 12.312 & 35.162 & 26.030 \\
\hline $6: R L$ & 64.473 & 12.327 & 30.141 & 15.632 & 12.312 & .000 & 23.610 & 16.052 \\
\hline 7:PV & 48.203 & 24.565 & 52.038 & 30.997 & 35.162 & 23.610 & .000 & 3.108 \\
\hline 8:LVA & 52.553 & 17.960 & 40.080 & 23.587 & 26.030 & 16.052 & 3.108 & .000 \\
\hline 9:LVA & 50.756 & 19.370 & 41.571 & 37.503 & 38.382 & 20.879 & 14.049 & 10.784 \\
\hline 10:PVA & 45.984 & 18.699 & 42.765 & 35.797 & 35.325 & 29.808 & 17.882 & 12.966 \\
\hline
\end{tabular}

${ }^{1}$ PVA (Argissolos Vermelho-Amarelo); ${ }^{2} \mathrm{RL}$ (Neossolos Litólicos); ${ }^{3} \mathrm{TC}$ (Luvissolos Crômicos); ${ }^{4} \mathrm{PV}$ (Argissolos Vermelhos); e

${ }^{5}$ LVA (Latossolos Vermelho-Amarelo).

Posteriormente foi analisado a variação temporal dos dados em estudo e o quanto eles são homogêneos. Nessa analise foi identificado quatro grupos homogêneos (Figura 4). Grupo 1: agrupou as umidades e profundidade do solo; Grupo 2: Precip6,
Precip11, Precip13 e Precip16; Grupo 4: Precip1 e Precip8 e Grupo 5: elevação do terreno e Precip3. Nota-se que a umidade do solo não se agrupou com a precipitação. A elevação do terreno se agrupo com a precipitação. Ou seja, sugere cautela avaliar a umidade 
do solo com a precipitação. Segundo Galvincio e França (2014), a umidade do solo depende principalmente da frequência e intensidade da precipitação e menos do total precipitado.
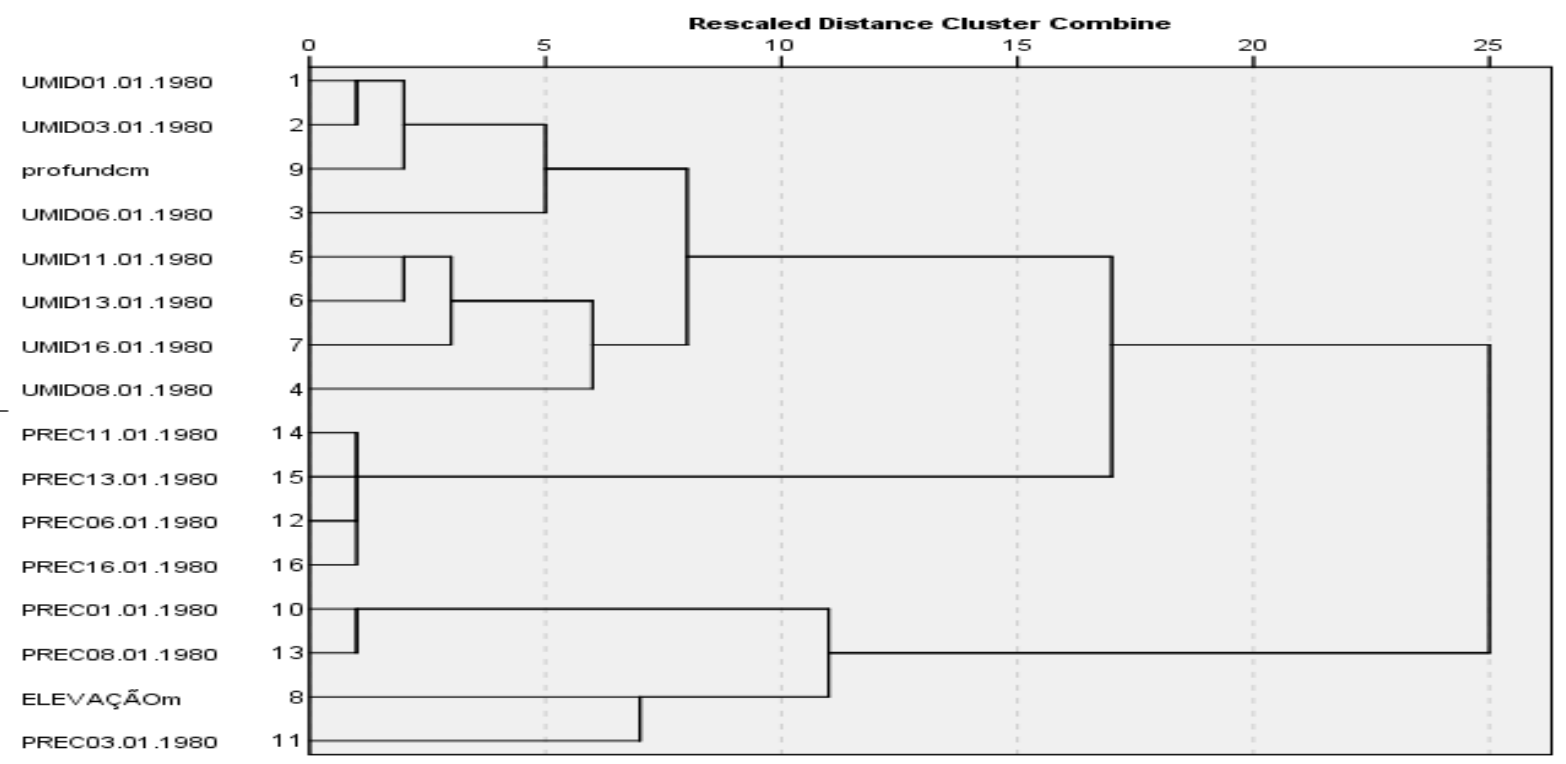

Figura 4 - Dendrograma pelo método de Ward da relação temporal entre as variáveis umidade do solo, precipitação, elevação do terreno e profundidade do solo.

Para verificar o quanto distante estão os grupos formadas da variação temporal das variáveis estudadas analisou a matriz de proximidade pela distância euclidiana ao quadrado (Tabelas 2, 3, 4 e 5). Nota-se que as distâncias da precipitação que mais se aproxima da umidade do solo é o da precipitação do dia 06/01 com a umidade do solo dos dias os dias 11 , 13 e 16 de janeiro de 1980.

Tabela 2 - Matriz de proximidade pela distância euclidiana ao quadrado para umidade nos dias 1, 3, 6 e 8 de janeiro de 1980.

\begin{tabular}{|c|c|c|c|c|}
\hline \multirow[t]{2}{*}{ DADOS } & \multicolumn{4}{|c|}{ MATRIX FILE INPUT } \\
\hline & $\begin{array}{c}\text { UMID } \\
01.01 .1980\end{array}$ & $\begin{array}{c}\text { UMID } \\
03.01 .1980\end{array}$ & $\begin{array}{c}\text { UMID } \\
06.01 .1980\end{array}$ & $\begin{array}{c}\text { UMID } \\
08.01 .1980\end{array}$ \\
\hline UMID 01.01.1980 & .000 & .039 & 7.635 & 9.221 \\
\hline UMID 03.01.1980 & .039 & .000 & 7.968 & 9.485 \\
\hline UMID 06.01.1980 & 7.635 & 7.968 & .000 & 15.674 \\
\hline UMID 08.01.1980 & 9.221 & 9.485 & 15.674 & .000 \\
\hline UMID 11.01.1980 & 7.750 & 7.546 & 13.191 & 8.446 \\
\hline UMID 13.01.1980 & 6.495 & 6.369 & 8.304 & 11.399 \\
\hline UMID 16.01.1980 & 15.569 & 14.882 & 17.195 & 16.998 \\
\hline ELEVAÇÃO m & 15.065 & 15.498 & 20.877 & 16.170 \\
\hline PROFUND (cm) & 3.159 & 2.965 & 10.189 & 14.552 \\
\hline PREC 01.01.1980 & 27.382 & 26.923 & 25.773 & 26.048 \\
\hline PREC 03.01.1980 & 24.966 & 25.149 & 21.077 & 26.553 \\
\hline PREC 06.01.1980 & 11.550 & 11.072 & 13.195 & 11.830 \\
\hline PREC 08.01.1980 & 28.325 & 27.897 & 25.301 & 25.267 \\
\hline PREC 11.01.1980 & 11.887 & 11.362 & 12.895 & 13.541 \\
\hline PREC 13.01.1980 & 11.670 & 11.149 & 12.593 & 13.682 \\
\hline PREC 16.01.1980 & 14.384 & 13.966 & 12.600 & 11.340 \\
\hline
\end{tabular}


Tabela 3 - Matriz de proximidade pela distância euclidiana ao quadrado para umidade nos dias 11, 13 e 16 de janeiro de 1980, elevação e profundidade.

\begin{tabular}{|c|c|c|c|c|c|}
\hline \multirow[t]{2}{*}{ DADOS } & \multicolumn{5}{|c|}{ MATRIX FILE INPUT } \\
\hline & $\begin{array}{c}\text { UMID } \\
11.01 .1980\end{array}$ & $\begin{array}{c}\text { UMID } \\
13.01 .1980\end{array}$ & $\begin{array}{c}\text { UMID } \\
16.01 .1980\end{array}$ & $\begin{array}{c}\text { ELEVAÇÃO } \\
\text { m }\end{array}$ & $\begin{array}{c}\text { PROFUND } \\
(\mathrm{cm})\end{array}$ \\
\hline UMID 01.01.1980 & 7.750 & 6.495 & 15.569 & 15.065 & 3.159 \\
\hline UMID 03.01.1980 & 7.546 & 6.369 & 14.882 & 15.498 & 2.965 \\
\hline UMID 06.01.1980 & 13.191 & 8.304 & 17.195 & 20.877 & 10.189 \\
\hline UMID 08.01.1980 & 8.446 & 11.399 & 16.998 & 16.170 & 14.552 \\
\hline UMID 11.01.1980 & .000 & 4.210 & 5.959 & 21.170 & 7.747 \\
\hline UMID 13.01.1980 & 4.210 & .000 & 7.421 & 16.142 & 4.257 \\
\hline UMID 16.01.1980 & 5.959 & 7.421 & .000 & 27.114 & 10.934 \\
\hline ELEVAÇÃO m & 21.170 & 16.142 & 27.114 & .000 & 15.624 \\
\hline PROFUND (cm) & 7.747 & 4.257 & 10.934 & 15.624 & .000 \\
\hline PREC 01.01.1980 & 19.805 & 18.389 & 15.352 & 17.226 & 20.875 \\
\hline PREC 03.01.1980 & 33.158 & 30.620 & 25.397 & 19.324 & 26.244 \\
\hline PREC 06.01.1980 & 13.104 & 14.456 & 12.200 & 29.121 & 11.550 \\
\hline PREC 08.01.1980 & 19.348 & 17.932 & 14.585 & 18.050 & 21.916 \\
\hline PREC 11.01.1980 & 16.922 & 15.835 & 14.805 & 26.773 & 12.060 \\
\hline PREC 13.01.1980 & 17.581 & 15.862 & 15.581 & 25.826 & 12.122 \\
\hline PREC 16.01.1980 & 13.983 & 14.591 & 11.479 & 30.193 & 14.362 \\
\hline
\end{tabular}

Tabela 4 - Matriz de proximidade pela distância euclidiana ao quadrado para precipitação nos dias 1, 3, 6 e 8 de janeiro de 1980.

\begin{tabular}{lcccc}
\hline \multicolumn{1}{c}{ DADOS } & \multicolumn{4}{c}{ MATRIX FILE INPUT } \\
\cline { 2 - 5 } & PREC 01.01.1980 & PREC 03.01.1980 PREC 06.01.1980 & PREC 08.01.1980 \\
\hline UMID 01.01.1980 & 27.382 & 24.966 & 11.550 & 28.325 \\
UMID 03.01.1980 & 26.923 & 25.149 & 11.072 & 27.897 \\
UMID 06.01.1980 & 25.773 & 21.077 & 13.195 & 25.301 \\
UMID 08.01.1980 & 26.048 & 26.553 & 11.830 & 25.267 \\
UMID 11.01.1980 & 19.805 & 33.158 & 13.104 & 19.348 \\
UMID 13.01.1980 & 18.389 & 30.620 & 14.456 & 17.932 \\
UMID 16.01.1980 & 15.352 & 25.397 & 12.200 & 14.585 \\
ELEVAÇÃO m & 17.226 & 19.324 & 29.121 & 18.050 \\
PROFUND (cm) & 20.875 & 26.244 & 11.550 & 21.916 \\
PREC 01.01.1980 & .000 & 20.952 & 20.000 & .190 \\
PREC 03.01.1980 & 20.952 & .000 & 20.952 & 21.445 \\
PREC 06.01.1980 & 20.000 & 20.952 & .000 & 20.068 \\
PREC 08.01.1980 & .190 & 21.445 & 20.068 & .000 \\
PREC 11.01.1980 & 20.936 & 18.480 & 1.009 & 21.161 \\
PREC 13.01.1980 & 21.145 & 18.167 & 1.511 & 21.410 \\
PREC 16.01.1980 & 20.901 & 20.570 & .990 & 20.192 \\
\hline
\end{tabular}


Tabela 5 - Matriz de proximidade pela distância euclidiana ao quadrado para precipitação nos dias 11, 13 e 16 de janeiro de 1980.

\begin{tabular}{lccc}
\hline \multirow{2}{*}{ DADOS } & \multicolumn{3}{c}{ MATRIX FILE INPUT } \\
\cline { 2 - 3 } \cline { 3 - 4 } & PREC 11.01.1980 & PREC 13.01.1980 & PREC 16.01.1980 \\
\hline UMID 01.01.1980 & 11.887 & 11.670 & 14.384 \\
UMID 03.01.1980 & 11.362 & 11.149 & 13.966 \\
UMID 06.01.1980 & 12.895 & 12.593 & 12.600 \\
UMID 08.01.1980 & 13.541 & 13.682 & 11.340 \\
UMID 11.01.1980 & 16.922 & 17.581 & 13.983 \\
UMID 13.01.1980 & 15.835 & 15.862 & 14.591 \\
UMID 16.01.1980 & 14.805 & 15.581 & 11.479 \\
ELEVAÇÃO m & 26.773 & 25.826 & 30.193 \\
PROFUND (cm) & 12.060 & 12.122 & 14.362 \\
PREC 01.01.1980 & 20.936 & 21.145 & 20.901 \\
PREC 03.01.1980 & 18.480 & 18.167 & 20.570 \\
PREC 06.01.1980 & 1.009 & 1.511 & .990 \\
PREC 08.01.1980 & 21.161 & 21.410 & 20.192 \\
PREC 11.01.1980 & .000 & .064 & 1.557 \\
PREC 13.01.1980 & .064 & .000 & 2.012 \\
PREC 16.01.1980 & 1.557 & 2.012 & .000 \\
\hline
\end{tabular}

Para saber se existe defasagem entre a relação entre a precipitação e umidade do solo foi realizada uma correlação com atrasos (leg) 14 para tentar encontrar a relação entre a precipitação do passado com a umidade do solo futura. O coeficiente de correlação não ultrapassou 0.20 . Ou seja, existe uma baixa relação entre o total precipitado e a umidade do solo.

\section{Conclusões e sugestões}

Após aplicação dos métodos estatísticos foi possível perceber que a umidade do solo não depende fortemente da precipitação. Sugere-se cautela no uso das relações entre precipitação e umidade do solo. E que a ação antrópica e as variações de intensidade de frequência da precipitação são mais importantes para avaliar a umidade do solo do que somente o total precipitado seja ele diário, mensal ou anual.

\section{Agradecimentos}

Á Coordenação de Aperfeiçoamento de Pessoal de Nível Superior (CAPES) pela concessão da bolsa à primeira autora, ao CNPQ, pelas bolsas de produtividade em pesquisa da segunda autora, e aos Laboratórios de Sensoriamento Remoto e Geoprocessamento (SERGEO) e de Análises dos Recursos Naturais (LARN) da Universidade Federal de Pernambuco, pela estrutura e apoio nas atividades de pesquisa.

\section{Referencias}

EMBRAPA. Empresa Brasileira de Pesquisa Agropecuária, 2006. Centro Nacional de Pesquisa de Solos. Sistema Brasileiro de Classificação de Solos. 2. ed. EMBRAPA-SPI, Rio de Janeiro.

Fechine, J.A.L., Galvíncio, J.D., 2008. Agrupamento da precipitação mensal da bacia hidrográfica do rio Brigida-PE, através da multivariada. Revista Brasileira de Geografia Física 1, 39-46.

Galvíncio, J.D., L.M.A., 2014. Impact of the hydric reposition in soil on the agriculture in semi arid region. Journal of Hyperspectral Remote Sensing 4, 134-152.

Hair, J.F., Anderson, R.E., Tatham, R.L., Black, W.C., 2005. Análise Multivariada de Dados. 5. ed. Bookman, Porto Alegre.

Sartori, S.D., 2008. Aplicações de técnicas de análise multivariada em experimentos agropecuários usando o software R. Dissertação (Mestrado). Piracicaba, ESALQ.

White, R.E., 2005. Principles and Practice of Soil Science: the soil as a natural resource. 4th ed. Blackwell, Oxford.

Whiting, M.L., Ustin, S.L., Zarco-Tejad, P., PalaciosOrueta, A., Vanderbilt, V.C., 2006. Hyperspectral mapping of crop and soils for precision agriculture. Remote Sensing and Modeling of Ecosystems for Sustainability III, 62980B. doi: 10.1117/12.681289 